\title{
The Chain Spread of Online Knowledge Transfer Process and Equivalence
}

\author{
Jing Wei ${ }^{1, *}$, Junchao Feng ${ }^{2}$ and Ruixiao Song ${ }^{3}$ \\ ${ }^{1}$ College of Management, Nanjing University of Posts \& Telecommunications, Nanjing 210023, China \\ ${ }^{2}$ Foreign Language Department, Harbin University of Science and Technology, Harbin, 150080, China \\ ${ }^{3}$ Department of Economic and Management, Nanjing University of Aeronautics and Astronautics, Nanjing 210016, \\ China
}

\begin{abstract}
The theoretical basis and interest-driven online knowledge transfer mechanism for the chain of knowledge transfer are explored. According to the network structure and the spread state of network nodes, we identify the online knowledge transfer network communication mode. Through the dissemination of the network structure and knowledge transfer state of the network nodes, we identify the chain dissemination of knowledge transfer networks and other online properties and also provide theoretical guidance. At last, we explore alternative node spread in important nodes in the chain, so as to organize the transfer of knowledge and effective practice. We also put forward some advice to promote the behavior of knowledge transfer.
\end{abstract}

Keyword: BBS, Chain spread, Equivalence, Knowledge management, Knowledge transfer, Online network.

\section{INTRODUCTION}

In the market, competition deterrence generated by the product itself has been slowly eliminated, product diversification competitive advantage has been lost with the enhancement of competitors' "moderate imitate force". However, the behavior of the knowledge transfer process provides a reliable way for the organization to obtain the more sustainable competitive advantage.

When, problems are encountered in the organizational environment, the individual needs necessary information to solve the problems and then transfer the information to individuals' or organization's knowledge. Therefore, the "code" and "interpretation" constitute two important stages of the whole knowledge transfer chain process.

"Encoding" is a process of knowledge transfer from "grassroots" to "high-level" which transfers the knowledge from individual storage to the organizations'. While "interpretation" phase is the stage of transfer the organization's knowledge to the user knowledge (Garavelli et al., 2002) [1].

Thus, the effective knowledge transfer process should include two stages: first, the individuals (or other organizations) pass the knowledge to the organization; second, the individuals obtain that knowledge and solve specific problems. There are two kinds of organizational knowledge flow stream which are inflows and outflows. When the organizations cannot solve the current problems with their own knowledge, they should acquire new knowledge flows in order to adapt the current situation (Nonaka, 1994) [2-6].
Therefore, when the organizations not only stores knowledge, but also learn from it, a complete process of knowledge transfer can be formed (Sharon Watson, 2006) [5].

This paper is organized as follows. The second part introduces the social exchange in knowledge transfer. The third part deduces the equivalence measure of online knowledge transfer network. The conclusions are given in section 4 .

\section{SOCIAL EXCHANGE THEORY IN KNOWLEDGE TRANSFER}

"Knowledge Contribution" is the first step in the chain of knowledge transfer process which is a benefit act for the organization. Why will individuals or other organizations maintain this behavior? Or why would individuals involve themselves in such cooperative behavior with the absence of a formal return?

In fact, the Organizational knowledge transfer process is the process by which the teams or organizations affect the other individuals or organizations (Argote \& Ingram, 2000). According to the social exchange theory, unequal phenomenon may lead to the loss of social independence. In order to maintain the independence of social interaction, the individual requires strategic resources, alternative resources, and reducing the coercive power of individuals (Blau, 1964) [7]. And effective use of existing organization knowledge is the "effective incentives" to force the other organizations to provide information and knowledge. The knowledge transfer process begins from this "effective incentives". It's also the driving factor of the organizations (or individuals) knowledge transfer behavior [8]. 
When individuals' knowledge is insufficient, they need "foreign" knowledge to complete their work. If someone looks forward to obtain valid knowledge, they will attract and generate knowledge transfer process effectively. Therefore, the "effective incentives" can generate the motivation of knowledge transfer and also generate continuous chain process of knowledge transfer.

\section{INTEREST-DRIVEN MECHANISM IN KNOWL- EDGE TRANSFER}

Holtshouse (1998) proposed the concept of "knowledge flow": knowledge can flow between knowledge providers and knowledge demanders [9]. Johansson (2004) divided the knowledge flow into two types: the first type is based on the exchange; the second type is the switching spillovers of the knowledge transfer [10]. This paper discusses the knowledge flow based on the knowledge exchange. According to Hothouse, if the knowledge can't flow effectively within the organization, it cannot be effectively utilized. The "knowledge flow" is the selective "push" process for the knowledge providers, and the "knowledge flow" is the selective "pull" process for the knowledge demanders (Holtshouse, 1998) [9]. This paper draws Zuo Meiyun's "knowledge potential" point of view and promotes Holtshouse's "knowledge flow" concept to the organization's internal and external perspective [11].

Only when there are individual differences in information dissemination, can the online information data be transmitted. According to Zuo Meiyun (2004), the knowledge can be transferred from the low content ones to the high content ones. There are three aspects to determine the level of knowledge of the potential: the amount of knowledge, the quality of knowledge and the knowledge structure [12].

\section{EQUIVALENCE MEASURE OF THE KNOWL- EDGE TRANSFER CHAIN PROCESS}

After the recognition of nodes of the high and low potential benefits, what could we do to avoid the resignation hazards of individuals? We not only improve the incentives for human capital management organization factors identified outside, but also minimize the importance of individual's resignation. Through the dissemination of knowledge transfer chain in peer measure, explore alternative node chain propagation of important nodes.

The analysis of peer networks includes the following three "reciprocity": (1) structural equivalence analyzes whether an actor is replaced without changing the relationship between the structures of the network under conditions and other actors. (2) auto-morphism reciprocity concerns sub-graph nodes in the collection that does not destroy the structure of the network location under conditions and replace another set of nodes. (3) rules of reciprocity, analysis of network nodes and occupies the same position in the network nodes for others, such as relationships. Here the main analysis of knowledge transfer networks such as chain transfer, and recognition of different chain structure is irreplaceable in the network, so the main rules of the overall analysis of the structure and node are of peer-to-peer nature [13].

\subsection{Construction of Knowledge Transfer Network}

We take the real forum records of certain enterprise's BBS as the analytical master of the knowledge transfer network in this paper. This online knowledge transfer network is made up of posting individuals which connected with each other. We use $N=\{1,2, \mathrm{~L} i, \mathrm{~L} n\}$ to denote the individuals in the online knowledge transfer network. Individuals' line directions are decided by reply posting direction. We select the two departments' BBS Replies record as a key analytical content. The former is company's business units and the latter is the company's background support department. These two sectors' knowledge transfer amount is usually large compared to the other sectors, so it's relatively easy to render regularity exclude statistical error, and will be referred to simply as advice- 4 and the advice- 5 .

\subsection{Equivalence Measure of the Overall Structure}

The chain structure of the network relationship can be considered as the structure reciprocity if there is no change of the chain structure and properties when the two nodes' swap positions. The substance of the structure reciprocity is to explore the node consistency in different locations of the network.

\section{(1) Similarity Analysis of Cross-Sectional}

We introduce the concept of cross-section to analysis the degree of the two nodes' structure consistency. In the directed network, the interface of $j$ means the $i$-th row $j$-th column data in the connectivity matrix. This data reflects the relationship of the node $\mathrm{j}$ and other nodes. Therefore, the structure reciprocity of the two nodes turns into the crosssection consistency of the two nodes' matrix.

After doing the cross-section analysis of advice-4 and advice-5, we obtain the section similarity matrix (Figs. 1a, 2a) and clustering result (Figs. 1b, 2b).

According to Fig. (1c): (1) 33 nodes are divided into 22 categories in the similarity of the 1.00 level, nodes are divided into a 7, 27, 2, 29, 21, 32, 4, 31, 32, 10, 30 groups which has the similar action and with similarities in the structure of chain structure; (2) 3, and 9 have the similar properties with the similarity level of 0.850 ; in the similarity level of 0.802 , node 5 and 22 have similar properties; (3) at the similarity level of $0.215,21$ and 26 are combined with other nodes, indicating that the gap between nodes 21 and 26 are the maximum compared to the other nodes; (4) the $7^{\text {th }}$ node and the node No. 28, 18, 24, 25 combined into one category at the similarity level of 0.695 , description No. 25 nodes and the 7 th node has a chain propagation structural similarity at the 0.695 level. The results indicate that these nodes have the structural similarity at a high level of similarity. If these nodes exchange positions, they may not change the network structure. 
Structural Equivalence Matrix

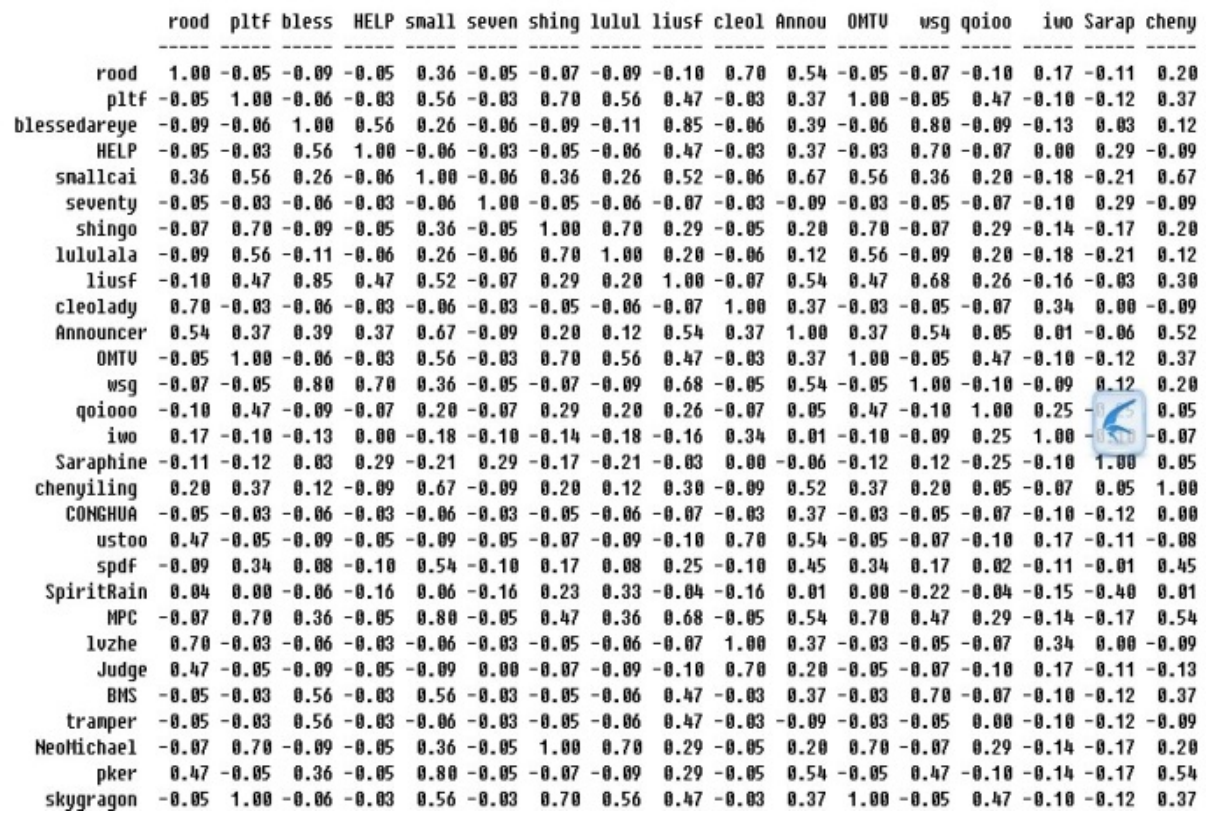

Fig. (1a). Advice - 4's similarity section matrix (part data).

HIERARCHICAL CLUSTERING OF EQUIUALENCE HATRIX

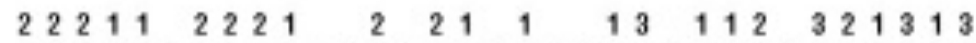

Level 160175258487729266393248941138053

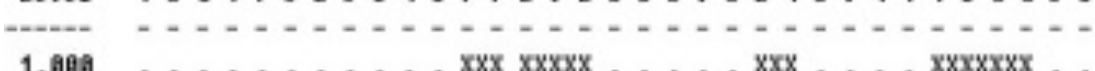

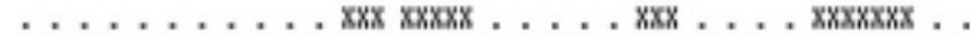

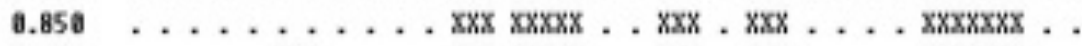

$0.882 \ldots x \mathrm{xxx} \ldots \mathrm{xxx} x \mathrm{xxxx} \ldots \mathrm{xxx}, \mathrm{xxx} \ldots \mathrm{xxxxxxy \ldots}$

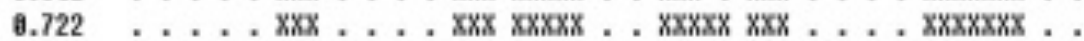

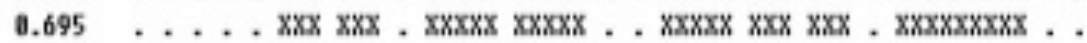

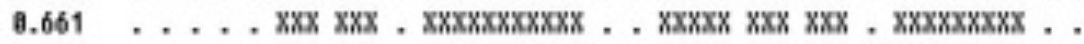

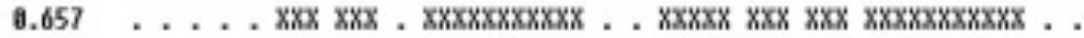

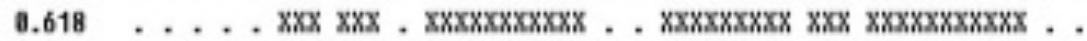

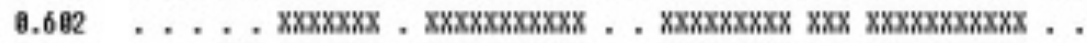

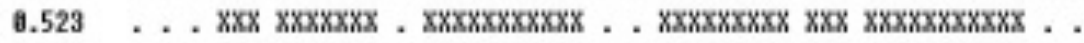

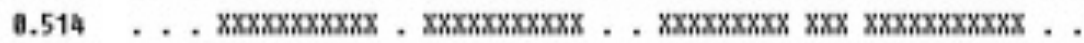

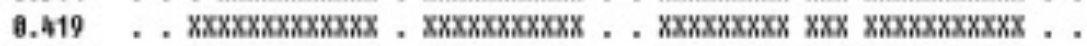

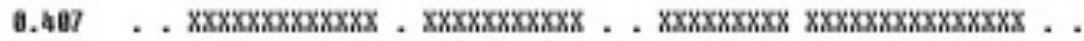

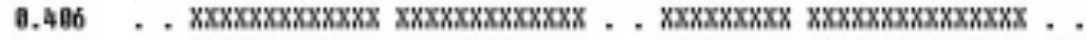

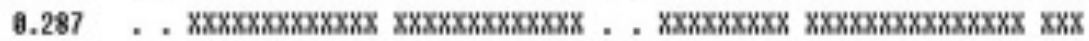

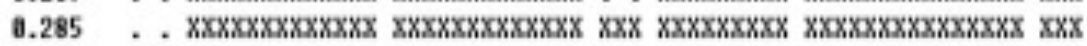

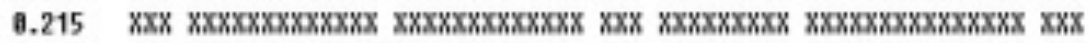

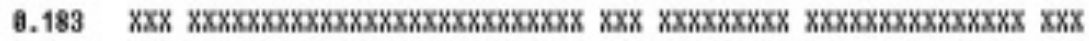

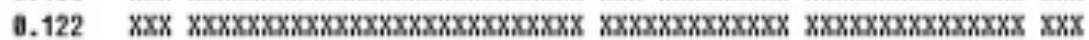

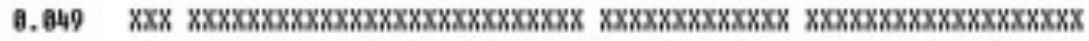

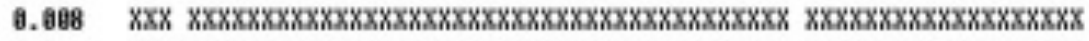

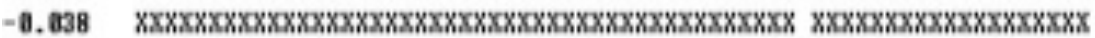

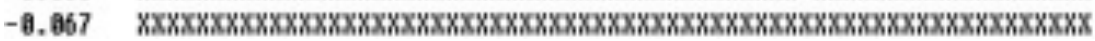

Fig. (1b). Advice - 4's structure clustering.

\section{(2) Multidimensional Scaling Description of the Cross- Section Similarity}

Multidimensional scaling is the method of expressing the node similarity in the two-dimensional map. Thus, between the nodes we can see the visual similarity of chain propagation, and the clustering information can be obtained on different dimensions.
Fig. (2a, b, c) show the data points with great similarity, which is similar to the analysis result of the closer in fig multidimensional scale cross-sectional. The number of nodes in advice- 4 is 32 , and the actual pressure index obtained is 0.045 . The number of nodes in advice- 5 is 18 , and the actual pressure index obtained is 0.008 which is less than the value of the experience. The degree of fitness is good. 


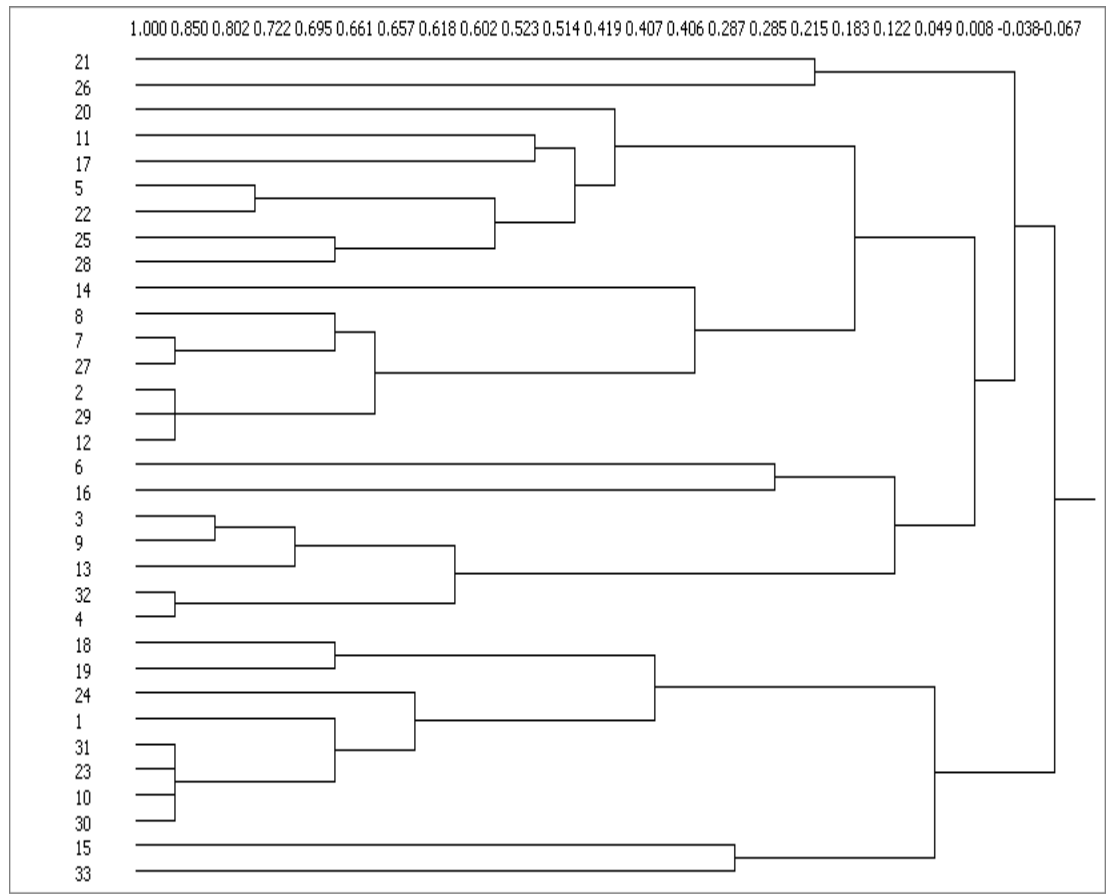

Fig. (1c). Advice - 5's clustering tree.

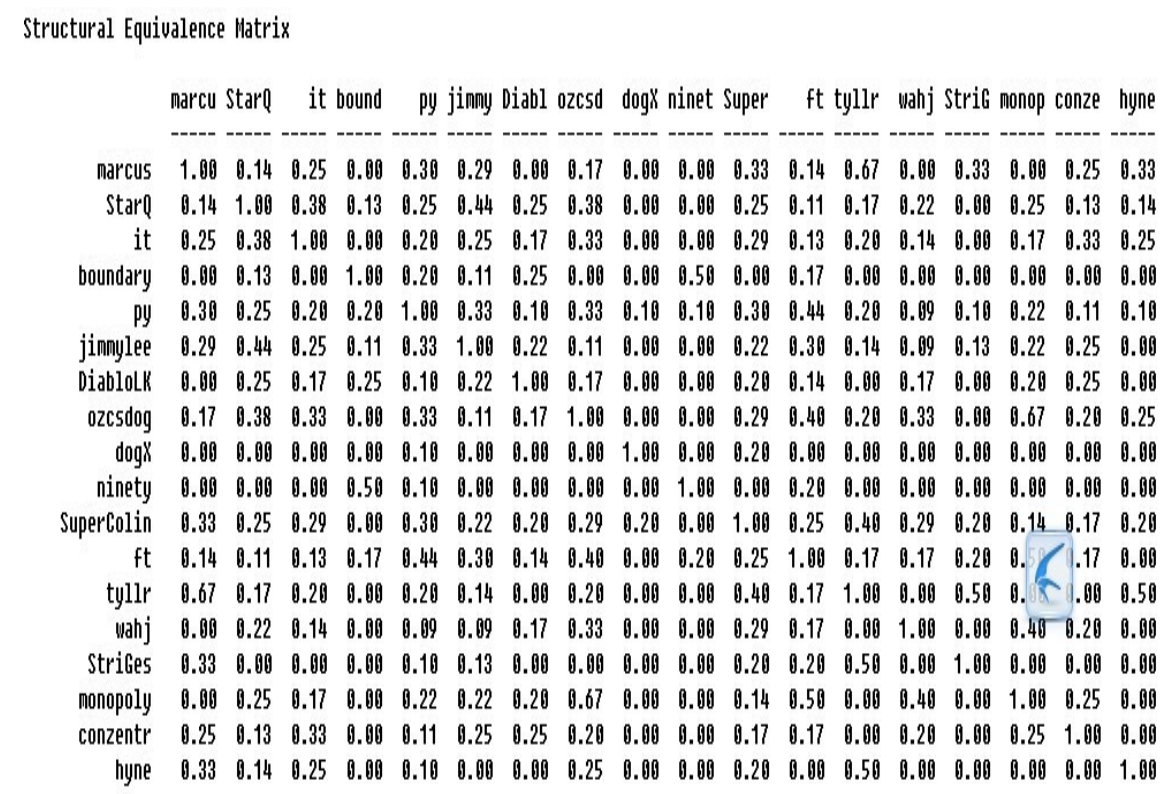

Fig, (2a). Advice - 5's similarity section matrix (part data).

As can be seen from the above analysis, advice-4 has the greater similarity compared to advice-5, i.e. the nodes' sectional similarity in advice-4 is higher, and the interchangeability between nodes chain is also much greater.

\subsection{Equivalence Measure of the Node Rules}

Equivalence of the node rules describes the same type of network communication node in the network chain structure. It also refers to the relationship of some nodes and the other nodes with respect to the same position. There are two REGE algorithms to measure the node rules equivalence: algorithms given class REGE algorithm and continuity REGE algorithm. Advice-4 and advice-5 are assigned directed networks, so we choose the continuity REGE selection algorithm for analysis.

\section{(1) Continuity REGE Algorithm}

We put the betweenness centrality and degree centrality of advice- 4 and advice-5 together. The degree centrality is used to measure the transact capacity of the nodes in the network and the betweenness centrality is used to measure the node's control ability. We can use it to analysis the interval distribution in Fig. (3a, b). 
HIERARCHICAL CLUSTERING OF EQUIUALENCE MATRIX

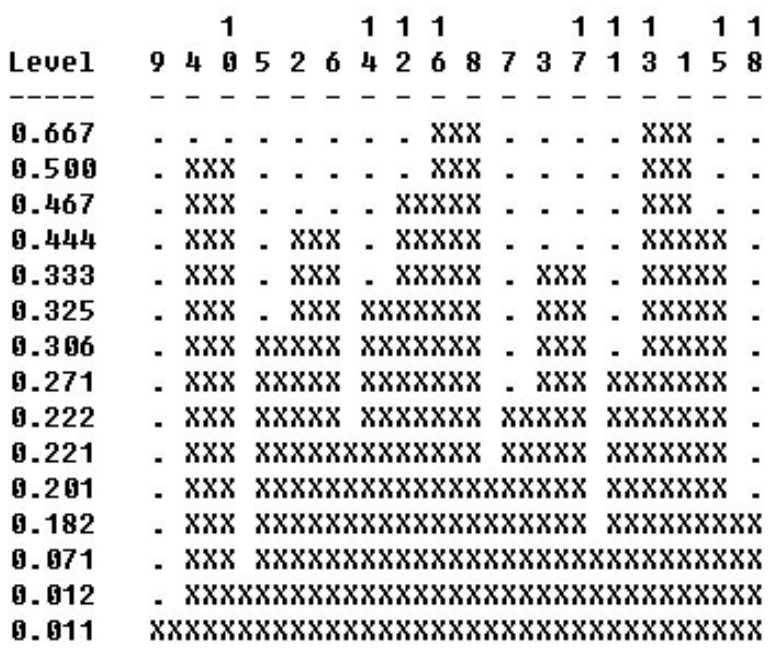

Fig. (2b). Advice - 5's structure clustering.

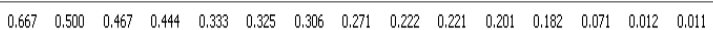

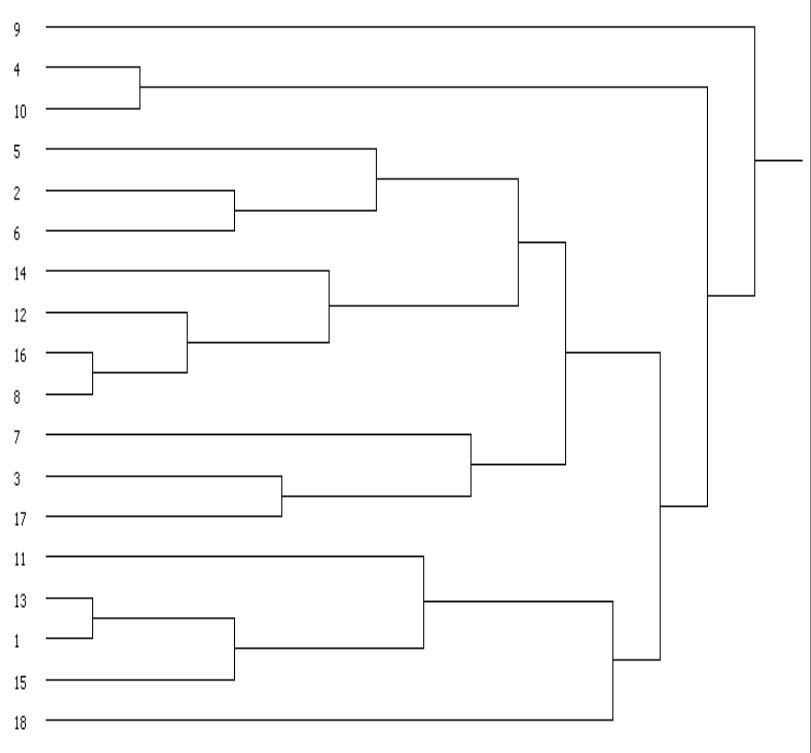

Fig. (2c). Advice -5 's clustering tree.

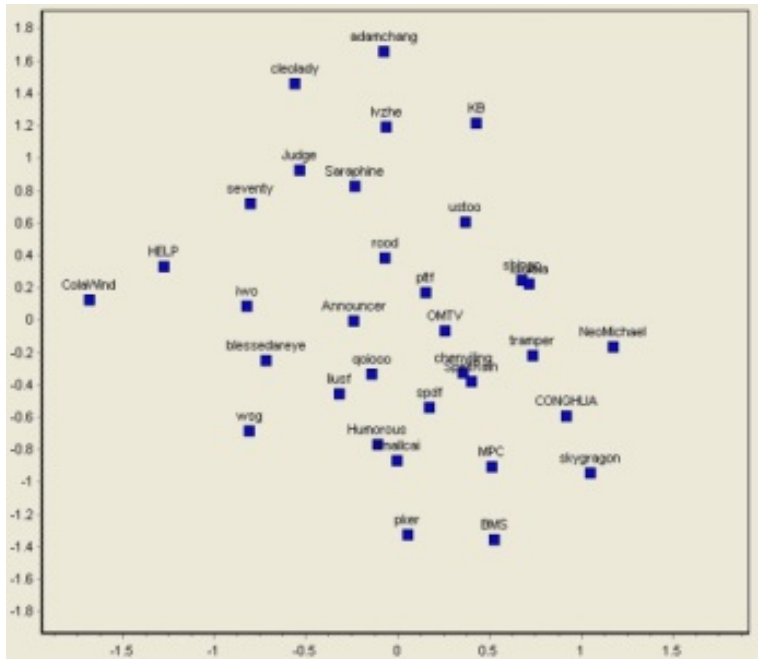

Fig. (3a). Advice - 4's multidimensional scaling analysis. 


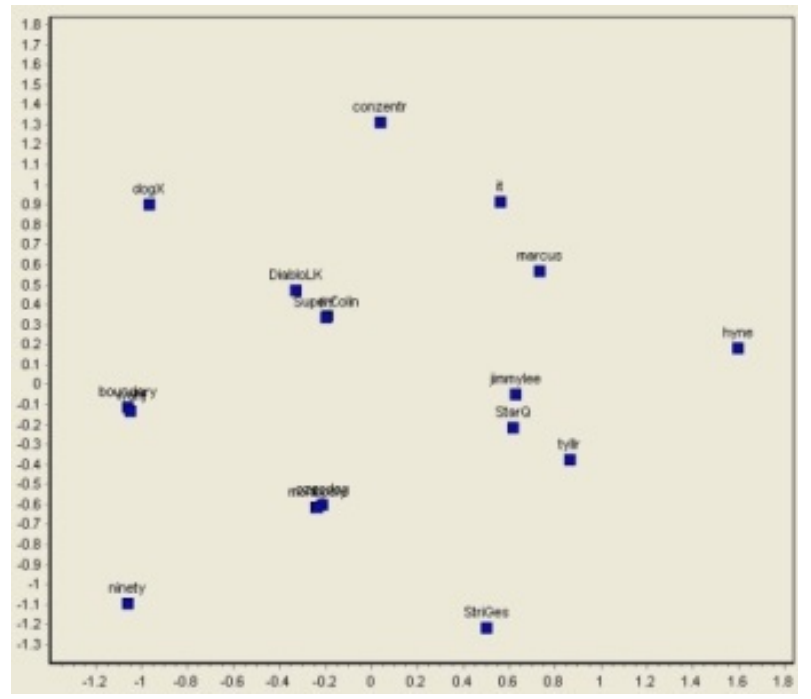

Fig. (3b). Advice - 5's multidimensional scaling analysis.

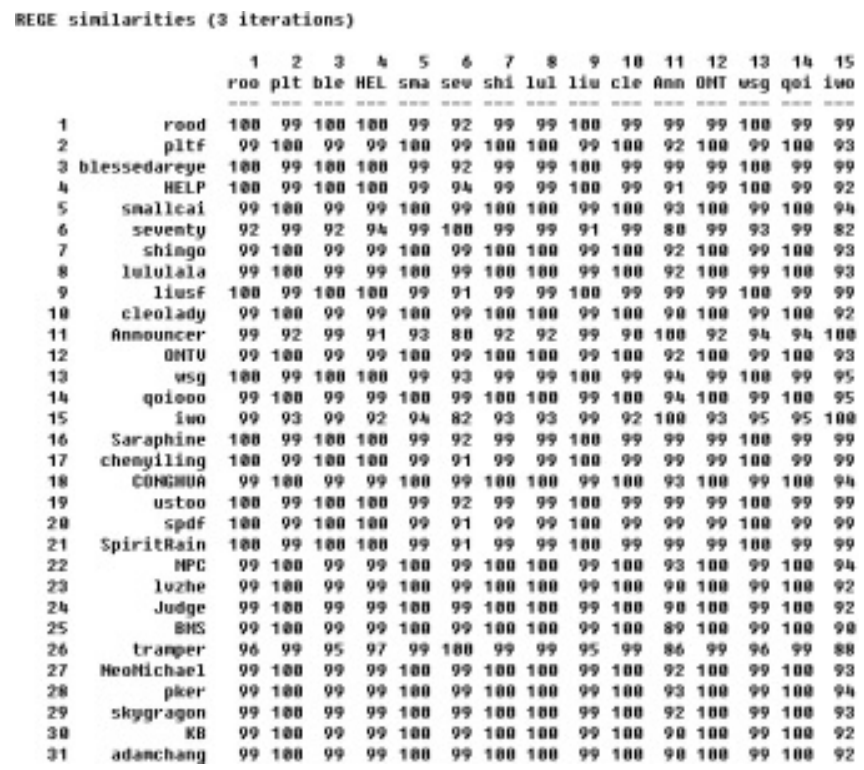

Fig. (4a). Advice - 4's similarity matrix of rules reciprocity (part data).

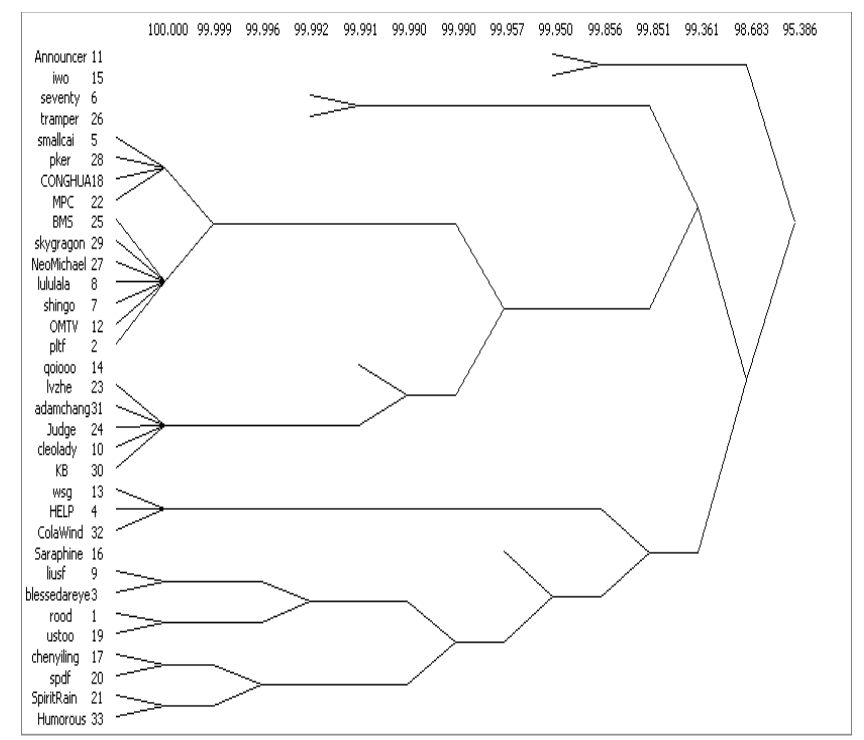

Fig. (4b). Advice -4 's clustering tree of rules reciprocity. 


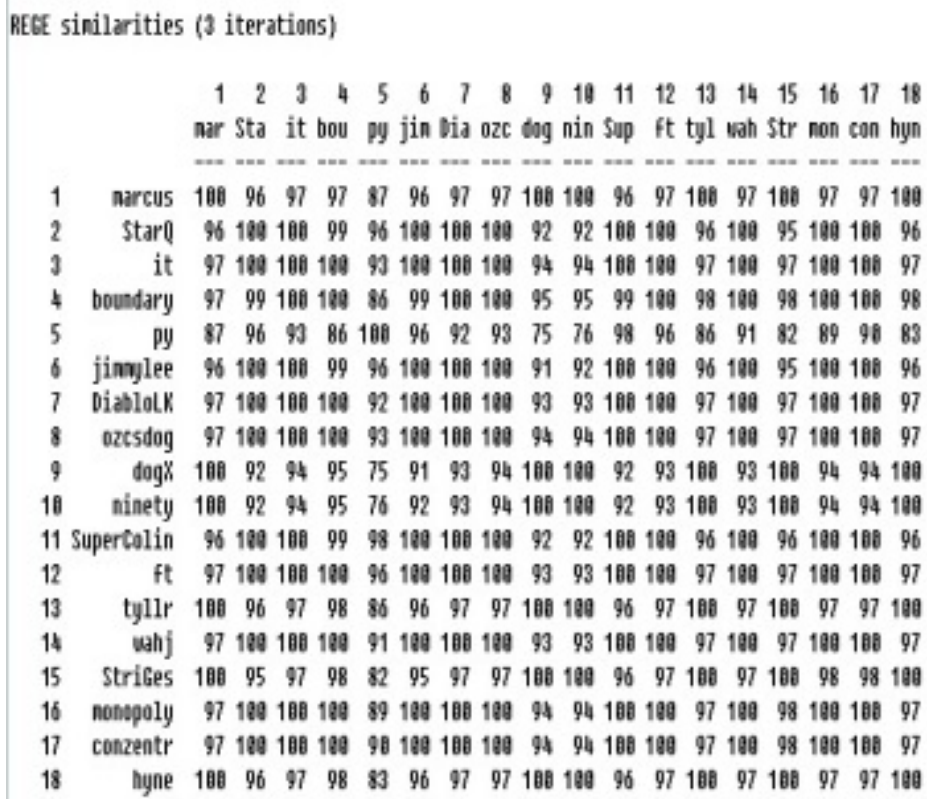

Fig. (5a). Advice - 5's similarity matrix of rules reciprocity (part data).

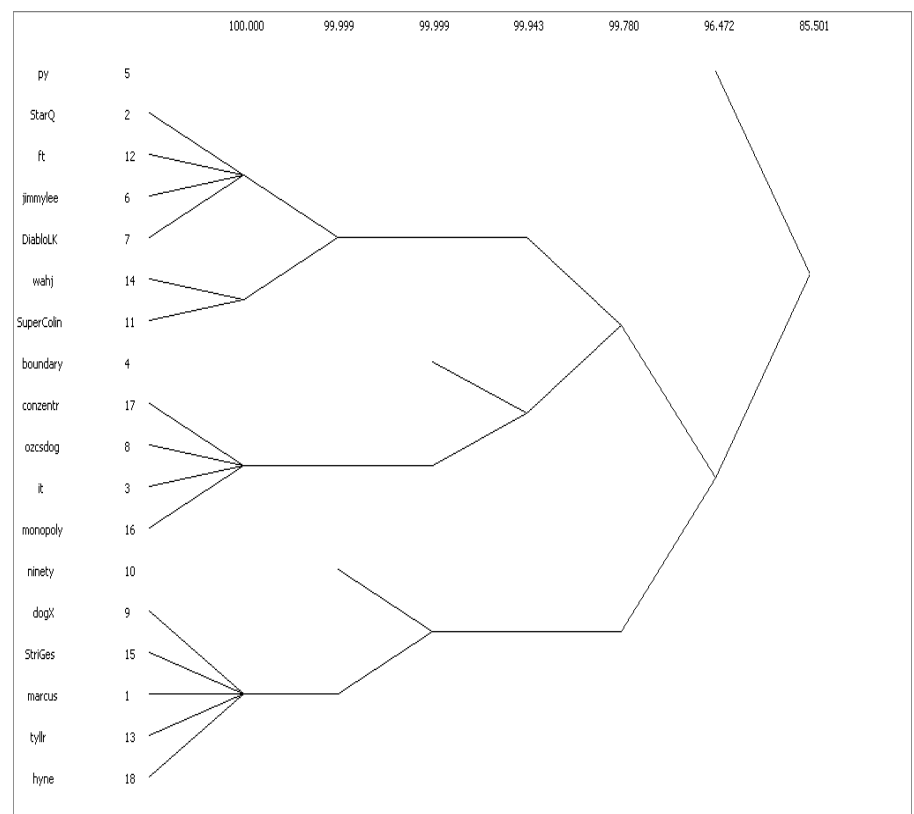

Fig. (5b). Advice -5 's clustering tree of rules reciprocity.

First, compute the node similarity matrix. And then, measure the degree to which the node "matches" the other ones. The greater is the degree, the more of the nodes reciprocity.

Fig. (4a, b) shows that in the advice-4 networks: (1) 5, 28, $18,22,25,29$ has complete rules similarity with the similarity coefficient of $100 \%$; (2) the 6 th node and the 26 th node have the rules reciprocity on the $99.991 \%$ level; (3) the $1 \mathrm{st}$ and the 15 th node have the worst reciprocity on the level of $99.851 \%$.

Fig. (5a, b) shows that in the advice-5 network: (1) 2, 12, 6,7 nodes have the rules reciprocity on a level of $100 \%$; (2) node 5 and node 10 have the rules reciprocity at the level of $95 \%$.
According to the above analysis, we obtain the nodes with the REGE algorithm reciprocity, but we cannot obtain the clear distinction between the roles of the node chain propagation. The Tabu algorithm through block model should be used to define network nodes corresponding to the role of chain propagation.

\section{(2) Tabu Algorithm Analysis}

Tabu algorithm is mainly used to determine the role of nodes. The assignment matrix will be replaced by a network node 0-1 matrix, and produce 0 - Block and 1 - Block as far as possible. This algorithm requires the data acquisition process after several trials, in order to obtain a more appropriate grouping. 


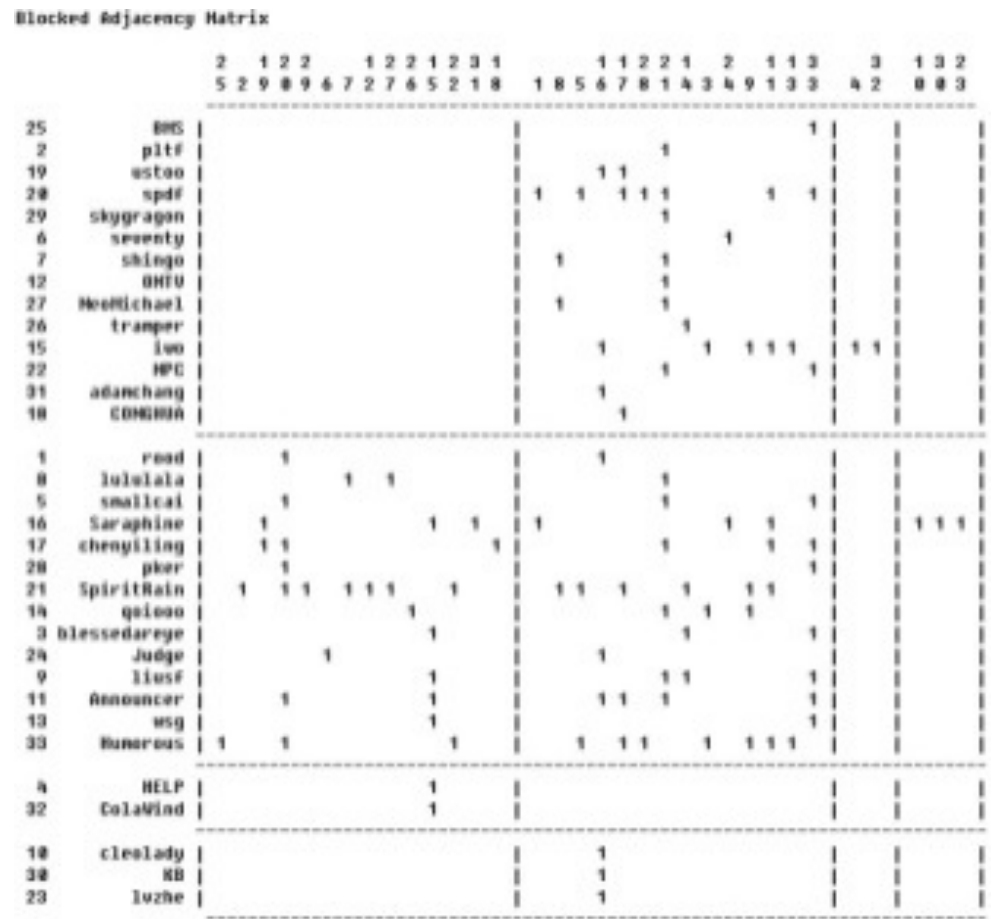

Fig. (6a). advice -4 's block matrix of Tabu algorithm.

\section{Blocked Adjacency Matrix}

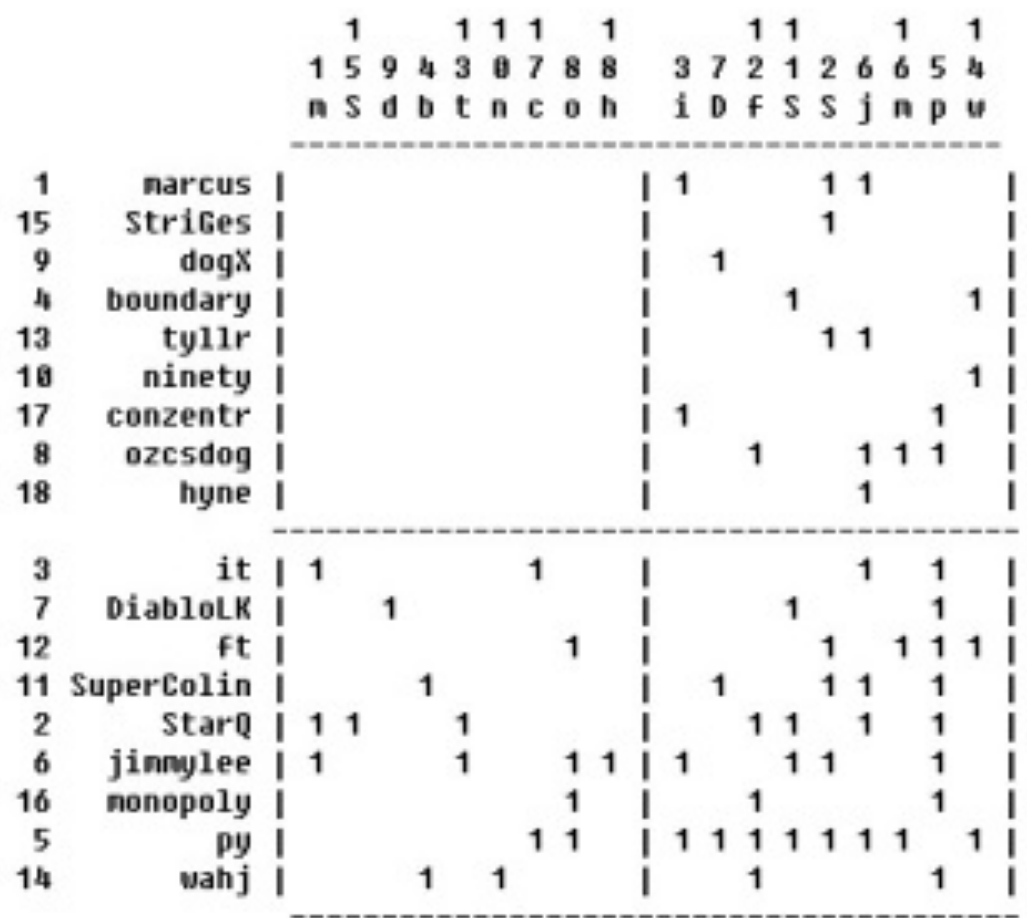

Fig. (6b). advice - 5's block matrix of Tabu algorithm.

After several trials of advice- 4 and advice-5, we get the block matrixes as shown in Figs. (6a and $6 \mathbf{6}$ ) which basically meets the 0 - Rule blocks - block 1 .

In advice-4, the nodes are divided into four groups: the first group has an "intermediary" role, and contacts with the second group; the second group is the core in the network which accepts and sends large amounts of information; with zero density the third and fourth group have both extremely non-active nodes in the chain propagation.

In advice-5, the nodes are divided into two groups: the nodes in the first group are chain-type transmission nodes which just accept the information from the second group; 
nodes in the second group both receive and accept information. They are active nodes in the network which have the highest density.

\section{CONCLUSION}

In this paper, we use the social exchange theory to revealing the theoretical foundation of the knowledge transfer process and interest-driven mechanism. After in-depth analysis, we use the actual network data to measure the reciprocity of the chain propagation process. Our aim is to explore alternative node in the chain propagation process through the reciprocity measurement. Once important nodes represent the organization; we can explore alternative individuals immediately to minimize the leave-damage, so as to provide valuable suggestions to the knowledge transfer to organizations.

\section{CONFLICT OF INTEREST}

The authors confirm that this article content has no conflict of interest.

\section{ACKNOWLEDGEMENTS}

This work was supported by the MOE (Ministry of Education in China) Project of Humanities and Social Sciences No. 13YJC630178, the Jiangsu Province Social Science Fund No.14TQC002, the Education Department of Jiangsu Province University Philosophy Social Science Fund Project No.2012SJB630050, Social science researches in Jiangsu Province No. 13SQC-106, Jiangsu Province Education Science "Twelfth Five Year Plan" key funding issues No. B$\mathrm{a} / 2013 / 01 / 021$, the Jiangsu provincial government scholarship program.

\section{REFERENCES}

[1] C. Garavelli, M. Gorgoglione and B. Scozzi, "Managing knowledge transfer by knowledge technologies", Technovation, vol. 22, pp. 269-279, 2002.

[2] I. Nonaka, "A dynamic theory of organizational knowledge creation", Organization Science, vol. 5, pp. 14-37, 1994.

[3] G. Hendrikse, Economics and Management of Organization: Motivation and Strategy, Mc Graw-Hill, USA, 2003.

[4] Z. Meiyun, Knowledge transfer and enterprise information, Science Press: Beijing, 2006.

[5] S. Watson, "How interdependence affects subsidiary performance", Journal of Business Research, vol. 59, pp.916-924, 2006.

[6] L. Argote, P. Ingram, J. M. Levine, and R. L. Moreland, "Knowledge transfer in organizations: learning from the experience of others", Organizational Behavior and Human Decision Processes, vol. 82, pp.1-8, 2002.

[7] P. M. Blau, Exchange and Power in Social Life, Wiley and Sons: New York, 1964

[8] S. Beechler, V. Pucik, J. Stephan and N. Campbe, "The transnational challenge: performance and expatriate presence in the overseas affiliates of Japanese MNCS", Advances in International Management, vol. 17, pp. 215-242, 2004.

[9] D. Holtshouse, "Knowledge research issues", California Management Review, vol. 40, pp. 277-280, 1998.

[10] B. Johansson, "Parsing the managerial of agglomeration and network externalies". CESIS Electronic Working Paper Series, Paper No. 02, 2004.

[11] Z. Meiyun, "Six types of knowledge transfer between the main enterprise information", Computer Systems \& Applications, vol.8, 2004.

[12] T.H Davenport and L. Prusak, Working knowledge: how organizations manage what they know: Harvard Business School Press, Boston, 1998.

[13] L. Jun, The whole network analysis: China Social Sciences Press, Beijing, 2009.

(C) Wei et al.; Licensee Bentham Open.

This is an open access article licensed under the terms of the Creative Commons Attribution Non-Commercial License (http://creativecommons.org/licenses/by-nc/3.0/) which permits unrestricted, non-commercial use, distribution and reproduction in any medium, provided the work is properly cited. 